\title{
Joint ESPGHAN/NASPGHAN Guidelines for the Management of Helicobacter pylori in Children and Adolescents (Update 2016)
}

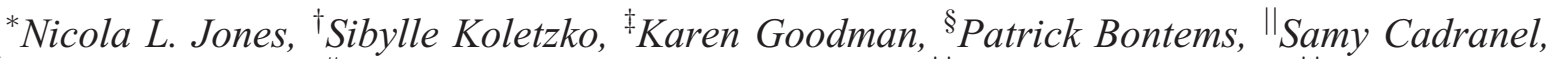 \\ "Thomas Casswall, " Steve Czinn, ${ }^{* *}$ Benjamin D. Gold, ${ }^{\dagger \dagger}$ Jeannette Guarner, ${ }^{\ddagger \dagger}$ Yoram Elitsur,

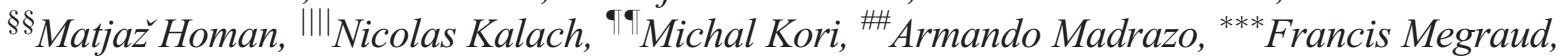 \\ ${ }^{\dagger \dagger}$ Alexandra Papadopoulou, and ${ }^{\ddagger \dagger}$ Marion Rowland, on behalf of ESPGHAN, NASPGHAN
}

\begin{abstract}
Background: Because of the changing epidemiology of Helicobacter pylori infection and low efficacy of currently recommended therapies, an update of the European Society for Paediatric Gastroenterology Hepatology and Nutrition/North American Society for Pediatric Gastroenterology, Hepatology and Nutrition recommendations for the diagnosis and management of $H$ pylori infection in children and adolescents is required.

Methods: A systematic review of the literature (time period: 2009-2014) was performed. Representatives of both societies evaluated the quality of evidence using GRADE (Grading of Recommendation Assessment, Development, and Evaluation) to formulate recommendations, which were voted upon and finalized using a Delphi process and face-to-face meeting.

Results: The consensus group recommended that invasive diagnostic testing for H pylori be performed only when treatment will be offered if tests are positive. To reach the aim of a $90 \%$ eradication rate with initial therapy, antibiotics should be tailored according to susceptibility testing. Therapy should be administered for 14 days, emphasizing strict adherence. Clarithromycin-containing regimens should be restricted to children infected with susceptible strains. When antibiotic susceptibility profiles are not known, high-dose triple therapy with proton pump inhibitor, amoxicillin, and metronidazole for 14 days or bismuthbased quadruple therapy is recommended. Success of therapy should be monitored after 4 to 8 weeks by reliable noninvasive tests.

Conclusions: The primary goal of clinical investigation is to identify the cause of upper gastrointestinal symptoms rather than $H$ pylori infection. Therefore, we recommend against a test and treat strategy. Decreasing eradication rates with previously recommended treatments call for changes to first-line therapies and broader availability of culture or molecular-based testing to tailor treatment to the individual child.
\end{abstract}

Key Words: adolescents, antibiotic susceptibility, ${ }^{13} \mathrm{C}$-urea breath test, children, eradication, Helicobacter pylori, triple therapy

(JPGN 2017;64: 991-1003)

Received July 4, 2016; accepted March 10, 2017.

From the $*$ Division of Gastroenterology, Hepatology and Nutrition, Cell Biology Program, Sickkids Toronto, Departments of Paediatrics and Physiology, University of Toronto, Toronto, Canada, the †Division of Gastroenterology and Hepatology, Dr. von Hauner Children's Hospital, Ludwig Maximilians University, Munich, Germany, the $\ddagger$ Department of Medicine and School of Public Health, Centre of Excellence for Gastrointestinal Inflammation and Immunity Research, University of Alberta, Edmonton, Alberta, Canada, the §Paediatric Gastroenterology Department, Hôpital Universitaire des Enfants Reine Fabiola, the ||Department of Paediatric Gastroenterology, Queen Fabiola University Children's Hospital, Université Libre de Bruxelles, Brussels, Belgium, the TPediatric Gastroenterology, Hepatology, and Nutrition, CLINTEC, Karolinska Institutet and Karolinska University Hospital, Stockholm, Sweden, the \#Department of Pediatrics, University of Maryland School of Medicine, Baltimore, MD, the $* *$ Children's Center for Digestive Healthcare, LLC, Gi Care for Kids, LLC, Children's Healthcare of
$H$ elicobacter pylori infection is acquired in childhood and remains an important cause of peptic ulcer disease (PUD) and gastric cancer (1). In comparison with adults, children and adolescents, however, infrequently develop these complications of infection. Furthermore H pylori infection in early childhood may be associated with immunologic benefits later in life (2).

The updated recommendations were extensively discussed in the light of decreasing efficacy of $H$ pylori eradication therapy in children and the rising prevalence of antibiotic-resistant strains. Although $H$ pylori infection is always associated with microscopic gastric inflammation, the overwhelming majority of $H$ pyloriinfected children are asymptomatic. Studies in children do not support a role for $H$ pylori infection in functional disorders such as recurrent abdominal pain (3). Therefore in children the decision to investigate and treat the infection should be supported by a clear benefit for the individual child.

There is renewed hope in the potential for primary prevention of infection based on the results of a recent vaccine study in Chinese children (4). Optimization of the vaccine strategy is, however, required to ensure long-lasting protection. Furthermore, additional studies demonstrating efficacy in different geographic regions are also needed before this vaccination strategy can be adopted in clinical practice.

The recommended goal for $H$ pylori treatment is an eradication rate of at least $90 \%$ to avoid further investigations and antibiotic use (5). First-line therapies recommended in previous guidelines have unacceptable treatment failures rates (6). Thus, based on the declining prevalence of the infection in some countries, and the ineffectiveness of previously recommended first-line eradication therapies, European Society for Paediatric Gastroenterology Hepatology and Nutrition (ESPGHAN) and North American Society for Pediatric Gastroenterology, Hepatology and Nutrition (NASPGHAN) determined that updated

Atlanta, the $\dagger$ † Department of Pathology and Laboratory Medicine, Emory University, Atlanta, GA, the $\ddagger \ddagger$ Department of Pediatrics, Gastroenterology Division, Marshall University School of Medicine, Huntington, WV, the $\S \S$ Faculty of Medicine, Department of Gastroenterology, Hepatology and Nutrition, University Children's Hospital Ljubljana, Ljubljana, Slovenia, the || $\mid$ Saint Antoine Pediatric Clinic, Saint Vincent de Paul Hospital, Groupement de l'Institut Catholique de Lille (GH-ICL), Catholic University, Lille, France, the $\boldsymbol{\top}^{\text {Kaplan Medical Center, Hadassah }}$ Medical School, Hebrew University, Jerusalem, Israel, the \#\#Pediatric Gastroenterology Division, Hospital de Pediatría, Centro Medico Nacional Siglo XXI, I.M.S.S. Mexico City, Mexico, the $* * *$ Laboratoire de Bactériologie, Université de Bordeaux, Bordeaux, France, the †† †ivision of Gastroenterology, Hepatology and Nutrition, First Department of Pediatrics, University of Athens, Children's Hospital "Ag. Sofia", Athens, Greece, and the $\ddagger \ddagger$ School of Medicine, University College Dublin, Dublin, Ireland. 
TABLE 1. Guideline recommendations

Synopsis of recommendations

1. We recommend that the primary goal of clinical investigation of gastrointestinal symptoms should be to determine the underlying cause of the symptoms and not solely the presence of $H$ pylori infection.

2a. We recommend that during endoscopy additional biopsies for RUT and culture should only be taken if treatment is likely to be offered if infection is confirmed.

2b. We suggest that if $H$ pylori infection is an incidental finding at endoscopy, treatment may be considered after careful discussion of the risks and benefits of $H$ pylori treatment with the patient/parents.

2c. We recommend against a "test and treat" strategy for $H$ pylori infection in children.

3. We recommend that testing for $H$ pylori be performed in children with gastric or duodenal ulcers. If $H$ pylori infection is identified then treatment should be advised and eradication be confirmed.

4. We recommend against diagnostic testing for $H$ pylori infection in children with functional abdominal pain.

5a. We recommend against diagnostic testing for $H$ pylori infection as part of the initial investigation in children with iron deficiency anemia.

$5 \mathrm{~b}$. We suggest that in children with refractory IDA in which other causes have been ruled out, testing for $H$ pylori during upper endoscopy may be considered.

6. We suggest that noninvasive diagnostic testing for $H$ pylori infection may be considered when investigating causes of chronic immune thrombocytopenic purpura (ITP).

7. We recommend against diagnostic testing for $H$ pylori infection when investigating causes of short stature.

8. We recommend that before testing for $H$ pylori, waiting at least 2 weeks after stopping proton pump inhibitor (PPI) and 4 weeks after stopping antibiotics.

9a. We recommend that the diagnosis of $H$ pylori infection should be based on either (a) histopathology (H pylori-positive gastritis) plus at least 1 other positive biopsy-based test or (b) positive culture.

9b. We recommend that for the diagnosis of $H$ pylori infection at upper gastrointestinal endoscopy, at least 6 gastric biopsies be obtained.

10. We recommend against using antibody-based tests (IgG, $\operatorname{IgA}$ ) for $H$ pylori in serum, whole blood, urine, and saliva in the clinical setting.

11. We recommend that antimicrobial sensitivity be obtained for the infecting $H$ pylori strain (s), and eradication therapy tailored accordingly.

12. We recommend that the effectiveness of first-line therapy be evaluated in national/regional centers.

13. We recommend that the physician explain to the patient/family the importance of adherence to the anti-H pylori therapy to enhance successful eradication.

14. We recommend first-line therapy for $H$ pylori infection as listed in Table 2.

15. We recommend that the outcome of anti-H pylori therapy be assessed at least 4 weeks after completion of therapy using one of the following tests. (a) The ${ }^{13} \mathrm{C}$-urea breath $\left({ }^{13} \mathrm{C}\right.$-UBT) test or (b) a 2 -step monoclonal stool antigen test.

16. We recommend that when $H$ pylori treatment fails, rescue therapy should be individualized considering antibiotic susceptibility, the age of the child, and available antimicrobial options.

$\mathrm{IDA}=$ iron deficiency anemia; $\mathrm{RUT}=$ rapid urease test.

guidelines for $H$ pylori infection in children and adolescents were required.

These guidelines apply only to pediatric patients, defined as children and adolescents below 18 years of age. Recommendations for children and adolescents may differ from recent guidelines for adults because of a different risk-benefit ratio depending on age and the fact that some antibiotics are not released or licensed for the pediatric population. The guidance in this document does not indicate an exclusive course of treatment or serve as a standard of medical care. Variations, taking into account individual and national circumstances, may be appropriate. This applies particularly to developing countries with high $H$ pylori infection rates in children and adolescents and with limited resources for health care.

\section{METHODS}

\section{Scope and Purpose}

Joint ESPGHAN/NASPGHAN guidelines were developed for the management of $H$ pylori infection in children and adolescents in 2009 and published in 2011 (6). Based on new clinical knowledge and in particular, increasing challenges with eradication therapy, NASPGHAN and ESPGHAN agreed to review the current literature to provide updated guidelines for the management of $H$ pylori infection in the pediatric population in North America and Europe.

\section{Literature Review}

A systematic literature search using PubMed, MEDLINE, EMBASE, Cochrane Library, and Scopus databases between October 1, 2009 to September 2014 using the following MESH terms (("Adolescent" [Mesh] OR “Child"'[Mesh] OR "Infant"'[Mesh] OR "Minors" [Mesh] OR "Pediatrics" [Mesh]) AND ("Helicobacter pylori”"[Mesh] OR “Helicobacter Infections"'[Mesh])) (K.G.). Studies were restricted to humans and conference abstracts and nonEnglish literature were excluded.

\section{Review, Grading of Evidence, and Consensus Process}

The consensus group consisted of an international group of experts including pediatric gastroenterologists, clinical
Address correspondence and reprint requests to Dr Sibylle Koletzko, MD, $\mathrm{PhD}$, Hauner Children's Hospital, Munich Medical Centre, Ludwig Maximilians University, Lindwurmstr. 4, 80337 Munich, Germany (e-mail: sibylle.koletzko@med.uni-muenchen.de).

This article has been developed as a Journal CME Activity by NASPGHAN. Visit http://www.naspghan.org/content/59/en/ContinuingMedical-Education-CME to view instructions, documentation, and the complete necessary steps to receive CME credit for reading this article.
Drs Jones and Koletzko contributed equally to this article

The authors report no conflicts of interest..

Copyright (c) 2017 by European Society for Pediatric Gastroenterology, Hepatology, and Nutrition and North American Society for Pediatric Gastroenterology, Hepatology, and Nutrition

DOI: 10.1097/MPG.0000000000001594 
epidemiologists, pathologists, and microbiologists. Each member of the consensus group provided disclosure of potential conflict of interest for 24 months prior using the societies' conflict of interest Web-based platforms. The consensus group was divided into 4 working groups (who to test, how to test, who to treat, and how to treat). Each working group reviewed the literature for their assigned topic and produced tables that rated the quality of evidence and strength of the recommendations using GRADE (Grading of Recommendation Assessment, Development, and Evaluation) (7). There were 2 levels of recommendations: strong or weak. The quality of evidence for each statement was graded as high, moderate, or low (A, B, and C). For most of the pediatric studies the evidence was low. In the absence of high-level evidence in the pediatric literature, studies in adults were considered and extrapolation performed, when appropriate. The tables and the referenced literature as full papers were made available to all authors on the Web-based guideline platform. The working groups developed initial recommendations based on the evidence. The entire group then voted anonymously on the initial statements using the Webbased platform. Suggestions for changes to the statements were incorporated and the statements were voted upon a second time before the face-to-face meeting.

In May 2015, a one-day, face-to-face meeting was held in Amsterdam. At the meeting, representatives from each of the groups presented the relevant data, the GRADE recommendations were reviewed, and inclusion and wording of the statements finalized. Lastly, the consensus group voted on their level of agreement with each of the statements. A statement was accepted if there was $>75 \%$ agreement. Per the GRADE system, "we recommend" was employed if the assigned strength of the recommendation was strong while "we suggest" was employed if the strength of the recommendation was weak or conditional.

\section{Manuscript}

Each of the working groups provided a summary of the written background evidence for the statements, which was then used to draft the initial manuscript by the co-chairs (N.L.J., S.K.). The manuscript was circulated to the consensus group for revisions and then to the members of ESPGHAN and NASPGHAN before submission for publication.

\section{Funding Sources}

ESPGHAN and NASPGHAN were the sole funding sources for the development of the guidelines.

\section{Recommendations}

Table 1 provides a synopsis of the new recommendations. Listed below are the individual recommendations, the GRADE rating, voting results, and a discussion of the supporting evidence. Practical practice points are included for each recommendation to aid the physician in management decisions for the individual patient.

Recommendation 1: We recommend that the primary goal of clinical investigation of gastrointestinal symptoms should be to determine the underlying cause of the symptoms and not solely the presence of $H$ pylori infection.

GRADE: This statement reflects consensus opinion on the philosophy of care for children rather than evidence. Therefore, a GRADE rating was not assigned. Agreement: $100 \%$.

\section{Practice Point}

1. In the absence of PUD herein defined as gastric or duodenal ulceration or erosions, eradication of $H$ pylori infection is not expected to result in improvement of symptoms.

\section{COMMENT:}

Since the last guidelines in 2009 (6), no new data support a role for $H$ pylori infection as a cause of symptoms in the absence of PUD. In cases of abdominal pain in which an organic cause is considered, a diagnostic upper endoscopy rather than noninvasive testing for $H$ pylori should be performed. This recommendation remains unchanged from the previous guidelines.

\section{Recommendation $2 a$ :}

We recommend that during endoscopy additional biopsies for rapid urease test (RUT) and culture should only be taken if treatment is likely to be offered if infection is confirmed.

GRADE: As there are no specific studies addressing this statement, no GRADE was assigned. Agreement: $86 \%$.

\section{Recommendation $2 b$ :}

We suggest that if $H$ pylori infection is an incidental finding at endoscopy, treatment may be considered following careful discussion with the patient/parents. GRADE: Weak recommendation. Quality of evidence: low. Agreement: $86 \%$.

\section{Practice Points}

1. If during endoscopy antral nodularity without mucosal lesions (gastric or duodenal erosions/ulcer) is visualized, biopsies for RUT and culture to diagnose $H$ pylori infection and guide treatment should only be taken by the endoscopist if treatment is likely to be offered upon confirmation of infection.

2. Upon offering anti-H pylori treatment to children without PUD (as outlined in Tables 2-4), physicians should explain that $H$ pylori infection is not likely to be the cause of the symptoms, and therefore H pylori treatment would not be expected to cure their symptoms. Discussion with parents or older children should include the potential risk of developing complications related to infection (PUD, gastric cancer) later in life weighed against the potential risks of treatment (eg, treatment failure, adverse effects of antibiotic use such as diarrhea, cramps, or negative alterations to the gut microbiome).

\section{COMMENT:}

Diagnostic investigation for $H$ pylori in children is justified only in cases in which the expected benefits outweigh the costs and risks of testing and subsequent treatment. Based on current evidence, treatment to eliminate $H$ pylori infection is not expected to improve symptoms in children, except in cases of PUD (2). Therefore, if peptic lesions are not clinically suspected or identified by endoscopy, invasive diagnostic testing for the sole purpose of detecting $H$ pylori infection should not be undertaken. 
TABLE 2. Recommended options for first-line therapy for $H$ pylori infection

\begin{tabular}{|c|c|}
\hline H pylori antimicrobial susceptibility & Suggested treatment \\
\hline \multicolumn{2}{|l|}{ Known } \\
\hline Susceptible to CLA and to MET & PPI-AMO-CLA 14d with standard dose* (Table 4) \\
\hline Resistant to CLA, susceptible to MET & PPI-AMO-MET $14 \mathrm{~d}$ or bismuth-based ${ }^{\dagger}$ \\
\hline Resistant to MET, susceptible to CLA & PPI-AMO-CLA $14 \mathrm{~d}$ or bismuth-based ${ }^{\dagger}$ \\
\hline Resistant to CLA and to MET & PPI-AMO-MET 14d with high dose for amoxicillin (Table 5) or bismuth-based $\mathrm{d}^{\dagger, \ddagger}$ \\
\hline \multicolumn{2}{|r|}{ - } \\
\hline & High-dose (Table 5) PPI-AMO-MET 14d or bismuth-based ${ }^{\dagger \neq}$ \\
\hline
\end{tabular}

$H$ pylori-associated gastritis may, however, be an incidental histopathologic finding during upper endoscopy performed for unrelated indications such as the diagnosis of inflammatory bowel disease, or celiac disease, especially in areas with a high prevalence of infection. The finding of $\mathrm{H}$ pylori-associated gastritis without duodenal or gastric mucosal lesions poses a dilemma for the pediatric gastroenterologist about whether to recommend eradication treatment. H pylori-associated gastritis without PUD, rarely gives rise to symptoms or progresses to more serious disease complications during childhood (2). The lower risk of complications in children may be in part explained by a different immune response toward the infection. In comparison with adults, gastric biopsies obtained from children infected with $H$ pylori show a decrease in inflammation. In addition, an increase in the number of immunosuppressive $\mathrm{T}$ regulatory (Treg) cells along with an antiinflammatory IL-10 instead of a proinflammatory IL-17 cytokine response is detected $(8,9)$.

In addition, in high-prevalence communities the reinfection rate in young children after successful eradication may be

\begin{tabular}{lccc}
\hline TABLE 3. Standard dosing regimen & & \\
\hline Drug & $\begin{array}{c}\text { Bodyweight } \\
\text { range }\end{array}$ & $\begin{array}{c}\text { Morning } \\
\text { dose, mg }\end{array}$ & $\begin{array}{c}\text { Evening } \\
\text { dose, mg }\end{array}$ \\
\hline PPI $^{*}$ & $15-24 \mathrm{~kg}$ & 20 & 20 \\
& $25-34 \mathrm{~kg}$ & 30 & 30 \\
Amoxicillin & $>35 \mathrm{~kg}$ & 40 & 40 \\
& $15-24 \mathrm{~kg}$ & 500 & 500 \\
& $25-34 \mathrm{~kg}$ & 750 & 750 \\
Clarithromycin & $>35-\mathrm{kg}$ & 1000 & 1000 \\
& $15-24 \mathrm{~kg}$ & 250 & 250 \\
& $25-34 \mathrm{~kg}$ & 500 & 250 \\
Metronidazole & $>35 \mathrm{~kg}$ & 500 & 500 \\
& $15-24 \mathrm{~kg}$ & 250 & 250 \\
Bismuth $^{\ddagger}$ & $25-34 \mathrm{~kg}$ & 500 & $250^{\dagger}$ \\
& $>35 \mathrm{~kg}$ & 500 & 500 \\
& $<10$ years & 262 QID & \\
\hline
\end{tabular}

PPI = proton pump inhibitor.

${ }^{*}$ The doses of the different proton pump inhibitors are not equivalent. See comment on PPI in recommendation 14.

'If oral suspension of metronidazole is employed, the dose can be divided to be equal every 12 hours.

${ }^{\ddagger}$ Bismuth in the United States and Canada comes as bismuth subsalicylate. significant. A study from Bolivia in a high-prevalence, low-income population showed a reinfection rate 1 year after successful therapy of $20 \%$ in children younger than 10 years, compared to $8 \%$ in older children and adolescents (10). These figures are higher than reinfection rates in adults patients living in high-prevalence countries in Latin America (11\% reinfection rate after 1 year) (11) or children and adolescents living in a low-prevalence country like Germany (2.3\% per year) (12).

The risk of $H$ pylori-associated cancer or mucosaassociated lymphoid tissue-lymphoma during childhood is extremely low in Europe and North America. The risk/benefit ratio may vary in different regions. In areas with a high rate of gastric cancer such as China or Japan, the benefits of treatment to reduce the risk of gastric cancer development may outweigh the risks of treatment (13). In Western countries there is epidemiologic evidence that $H$ pylori infection, especially in young children, may be associated with a reduced prevalence of allergic disease (14-18). Higher prevalence of H pylori infection may, however, be a surrogate marker for host demographics that could confer protection against autoimmunity and allergy. Therefore, although there is evidence for a causal relationship in mouse models of asthma and inflammation (18), prospective randomized intervention studies in humans are needed to demonstrate a causal relationship. There are currently no good biomarkers to identify the small number of individuals that will go on to develop more severe sequelae of infection later in life. Thus, the decision to treat $H$ pylori-associated gastritis in the absence of PUD when found as an incidental finding at upper endoscopy should be carefully discussed with the patient and family taking into consideration the potential risk and benefits of treatment in the individual patient.

Recommendation 2c: We recommend against a "test and treat" strategy for $H$ pylori infection in children. GRADE: Strong recommendation. Quality of evidence: low (indirect). Agreement: $100 \%$.

\section{Practice Points}

- The goal of clinical investigation is to detect the cause of the child's symptoms. Since current evidence indicates that $H$ pylori infection does not cause symptoms in the absence of $P U D$, performing a noninvasive test to detect infection and treat if the test is positive is not warranted. 


\begin{tabular}{lcc}
\hline \multicolumn{3}{l}{ TABLE 4. High dosing regimen for amoxicillin } \\
\hline Bodyweight range, $\mathrm{kg}$ & Morning dose, mg & Evening dose, mg \\
\hline $15-24$ & 750 & 750 \\
$25-34$ & 1000 & 1000 \\
$>35$ & 1500 & 1500 \\
\hline
\end{tabular}

\section{COMMENT:}

Since the last guidelines in 2009 , there is no evidence to support a "test and treat" strategy for H pylori infection in children. Therefore, this recommendation against noninvasive testing and treating in case of positive results remains unchanged from the previous guidelines.

Recommendation 3: We recommend that testing for $H$ pylori be performed in children with gastric or duodenal PUD. If $H$ pylori infection is identified then treatment should be administered and eradication confirmed.

GRADE: Strong recommendation. Quality of evidence: high. Agreement: $100 \%$.

\section{Practice Points}

- Although H pylori infection is only one of several causes of gastric or duodenal erosions/ulceration in children, it is a treatable condition. Eradication of infection prevents ulcer recurrence. If erosions, ulcers, or scarring are visualized during upper endoscopy, biopsies (as outlined in Recommendation 9) should be taken to identify the presence of $H$ pylori infection.

- Proton pump inhibitor (PPI) monotherapy may be continued after eradication therapy for another 2 to 4 weeks in patients with PUD.

- Successful H pylori eradication is associated with cure of PUD and very low risk of relapse. Therefore monitoring the success of therapy is mandatory in these patients 4 to 6 weeks after stopping antibiotics and at least 2 weeks after stopping PPI therapy. In case of eradication failure, the patient should receive rescue therapy as outlined in Table 5.

- Intake of acid-suppressive drugs and antibiotics decrease the sensitivity of all biopsy-based tests for $H$ pylori (see recommendation 8). Before endoscopy an accurate history for drug intake during the previous 4 weeks should be obtained from the child and caregivers.

- Active bleeding decreases the sensitivity of biopsy-based tests for infection.

- If false-negative results of invasive tests are suspected due to drug intake or active bleeding, noninvasive tests may be helpful to determine whether the ulcer may be related to $H$ pylori infection.

- Successful H pylori eradication is associated with long-term cure of PUD in children. Therefore monitoring the success of therapy 4 to 6 weeks after stopping therapy and re-treatment in case of failure is mandatory in these patients.

\section{COMMENT:}

Although the rates of $H$ pylori infection are declining, infection still remains an important cause of PUD (19). Furthermore, eradication of infection is associated with long-term cure of PUD in children.

In the previous joint guidelines, a weak recommendation for testing for $H$ pylori infection in the setting of a first-degree relative with gastric cancer was included (6). As there remains no evidence for this recommendation, and the question rarely arises in clinical pediatric gastroenterology practice, it was decided to remove this recommendation from the current guidelines.

\section{Recommendation 4}

We recommend against diagnostic testing for $H$ pylori infection in children with functional abdominal pain disorders.

GRADE: Strong recommendation. Quality of evidence: high. Agreement: 100\%.

\section{Practice Points}

- Children with recurrent abdominal pain without any alarm signs or symptoms most likely have functional pain independent of $H$ pylori status.

TABLE 5. Rescue therapies in pediatric patients who failed therapy

\begin{tabular}{|c|c|c|c|}
\hline & Initial antibiotic susceptibility & Past treatment regimen & Rescue treatment \\
\hline \multirow[t]{2}{*}{1} & $\begin{array}{l}\text { Clarithromycin and metronidazole } \\
\text { susceptible }\end{array}$ & $\begin{array}{l}\text { Triple therapy using amoxicillin and } \\
\text { clarithromycin }\end{array}$ & Triple therapy using amoxicillin and metronidazole \\
\hline & & $\begin{array}{l}\text { Triple therapy using amoxicillin } \\
\text { and metronidazole }\end{array}$ & Triple therapy using amoxicillin and clarithromycin \\
\hline 2 & $\begin{array}{l}\text { Clarithromycin and metronidazole } \\
\text { susceptible }\end{array}$ & Sequential therapy & $\begin{array}{l}\text { Consider performing a second endoscopy and use } \\
\text { a tailored treatment for } 14 \mathrm{~d} \text {; or treat like } \\
\text { double resistance (Table } 2)^{*}\end{array}$ \\
\hline 3 & Clarithromycin resistant & Triple therapy using metronidazole & Treat like double resistance (Table 2$)^{*}$ \\
\hline 4 & Metronidazole resistant & Triple therapy using clarithromycin & $\begin{array}{l}\text { Consider performing a second endoscopy and use a } \\
\text { tailored treatment for } 14 \text { days or treat like double } \\
\text { resistance (Table } 2)^{*}\end{array}$ \\
\hline 5 & $\begin{array}{l}\text { Primary antimicrobial } \\
\text { susceptibility unknown }\end{array}$ & Triple therapy or sequential therapy & $\begin{array}{l}\text { Consider performing a second endoscopy to assess } \\
\text { secondary antimicrobial susceptibility; or treat like } \\
\text { double resistance (Table 2) }\end{array}$ \\
\hline
\end{tabular}

\footnotetext{
*In adolescents levofloxacin or tetracycline may be considered.
} 
- Alarm signs include persistent right upper or right lower quadrant pain, dysphagia, odynophagia, persistent vomiting, gastrointestinal blood loss, involuntary weight loss, deceleration of linear growth, delayed puberty, unexplained fever, and a family history of inflammatory bowel disease, celiac disease, or PUD (20).

- A positive noninvasive test may induce anxiety in children with functional pain or their parents with the consequence of referral for upper endoscopy. Therefore, diagnostic testing for the infection should not be undertaken in these children.

\section{COMMENT:}

Treatment to eliminate $H$ pylori infection is not expected to improve symptoms in children, except in cases of PUD. Therefore, in children fulfilling the Rome criteria for functional abdominal pain diagnostic testing (noninvasive or invasive) for $H$ pylori infection should not be undertaken (21-26). In the absence of alarm signs or symptoms, recent updated recommendations from the committee for ROME IV did not identify compelling evidence to support upper endoscopy as part of the diagnostic work up (20), discussed in comments section for recommendations $2 \mathrm{a}$ and $2 \mathrm{~b}$.

\section{Recommendation 5 a}

We recommend against diagnostic testing for $H$ pylori infection as part of the initial investigation in children with iron deficiency anemia (IDA).

GRADE: Strong recommendation. Quality of evidence: moderate. Agreement: $93 \%$.

Recommendation $5 b$

We suggest that in children with refractory IDA in which other causes have been ruled out, testing for $H$ pylori during upper endoscopy may be considered. GRADE: Weak recommendation. Quality of evidence: low. Agreement: $100 \%$.

\section{Practice Points}

1. A diagnosis of IDA is made when both anemia and iron deficiency are present.

2. Children with IDA should be managed according to current guidelines for the treatment of IDA considering the clinical history and age of the child.

3. Noninvasive testing for $H$ pylori is not recommended as part of the initial investigation of IDA in children.

4. If upper endoscopy is clinically indicated in the management of IDA refractory to iron therapy, biopsies for the diagnosis of $H$ pylori as outlined in recommendation 9 may be considered.

5. If H pylori infection is detected in the setting of refractory IDA, eradication therapy for $H$ pylori should be combined with iron supplementation.

6. Noninvasive testing for $H$ pylori in the case of refractory IDA is not recommended.

\section{COMMENT:}

Children are at particular risk of IDA during the first 5 years of life because of their increased iron requirements during periods of rapid growth (27). More than 300 million preschool and schoolage children worldwide are anemic due to iron deficiency (28). The risk factors for IDA and $H$ pylori are similar with many children having concomitant IDA and $H$ pylori infection $(29,30)$. There is, however, no causal relationship between IDA and $H$ pylori. Since the last guidelines a number of methodologically well-conducted studies have examined the relationship between iron deficiency/ IDA and $H$ pylori, and all conclude that there is no evidence for a causal relationship between IDA and $H$ pylori (31-34).

The possibility that IDA may be due to blood loss associated with PUD, or iron scavenging by the bacteria (35) must be considered in children with refractory IDA who have not responded to initial iron supplementation therapy, rapidly relapse or who have other alarm symptoms (34). If there are clinical indications for upper endoscopy it is suggested that biopsies to confirm or rule out the presence of $H$ pylori infection may be considered. Since the publication of the previous guidelines there are no studies that specifically examine the relationship between refractory IDA and $H$ pylori. One study (36) that examined the effect of $H$ pylori eradication on refractory IDA was excluded because it did not meet the inclusion criteria. Thus this recommendation remains unchanged from previous guidelines.

\section{Recommendation 6}

We suggest that noninvasive diagnostic testing for $H$ pylori infection may be considered when investigating causes of chronic immune thrombocytopenic purpura (ITP).

GRADE: Weak recommendation. Quality of evidence: low. Agreement: 93\%.

\section{Practice Points}

1. In the patient with chronic ITP (cITP) and thrombocytopenia, noninvasive testing to diagnose the presence of infection should be employed. If the noninvasive test is positive it has to be decided on an individual basis and depending on the platelet count whether an upper endoscopy is needed before eradication therapy.

\section{COMMENT:}

cITP (previously idiopathic thrombocytopenic purpura) in childhood is an autoimmune disease with platelet autoantibody formation resulting in platelet destruction and thrombocytopenia (platelet count $<100 \times 10^{9} / \mathrm{L}$ ) for at least 12 months before presentation. Since the last guidelines 2 small intervention studies reported results of treating $H$ pylori infection in the setting of ITP in children $(37,38)$. In both of these studies, an improvement in platelet count occurred in a proportion of patients following $H$ pylori eradication. These studies were, however, nonrandomized and included small numbers of patients with a short follow-up time. In a small, randomized controlled trial of 22 children with $H$ pylori infection and cITP, increases in platelet counts were detected in more children in the anti-H pylori treatment group; however the follow-up period was short (39). Thus based on these limited studies, a weak recommendation for testing and treating for $H$ pylori infection in the patient with cITP was included in the guidelines. Additional well-designed interventional studies are, however, required to provide support for this recommendation.

\section{Recommendation 7}

We recommend against diagnostic testing for $H$ pylori infection when investigating causes of short stature. GRADE: strong recommendation. Quality of evidence: moderate. Agreement: $79 \%$ 


\section{COMMENT:}

The hypothesis that eliminating H pylori infection in children will prevent growth deficits has been investigated in few adequately designed studies. A few new cross-sectional studies add to the literature on associations between $H$ pylori infection and anthropometric indicators in children (40-44), with inconsistent results across studies. Epidemiological, observation or case-control studies are not designed to prove causation. Furthermore, both $\mathrm{H}$ pylori infection and short stature are associated with low socioeconomic status including poor nutrition. The only well-designed intervention study in the current review period was performed in children in the Colombian Andes in a setting of poor hygiene and high prevalence of both malnutrition and chronic infections $(45,46)$. Thus, further research is needed to assess the effect of offering treatment for $H$ pylori in children with short stature in a North American or European setting.

\section{Recommendation 8}

We recommend that before testing for $H$ pylori, wait at least 2 weeks after stopping PPIs and 4 weeks after stopping antibiotics.

GRADE: Strong recommendation. Quality of evidence: low. Agreement: 100\%.

\section{Practice Points}

1. Parents and guardians should be asked about drug intake during the 4 weeks before testing.

2. These time intervals apply for primary testing to diagnose or exclude $H$ pylori infection and to monitor treatment success. It applies for both invasive and noninvasive (urea breath test [UBT], stool antigen) testing.

3. If acid suppressive therapy cannot be discontinued for 2 weeks because of recurrence of symptoms, changing to an H2-receptor antagonist with discontinuation of the drug 2 days before testing may improve the sensitivity of the diagnostic test.

4. Antibiotics may suppress bacterial growth and may result in false-negative test results in all applied diagnostic methods except serology (which is not recommended).

\section{COMMENT:}

PPI therapy may give false-negative diagnostic test results for $H$ pylori infection due to suppression of $H$ pylori replication (47). No additional studies have been published since the last guidelines; thus, these recommendations remain the same as the previous guidelines.

\section{Recommendation $9 a$}

We recommend that the diagnosis of H pylori infection should be based on either: positive culture or $H$ pylori gastritis on histopathology with at least 1 other positive biopsy-based test.

GRADE: Strong recommendation. Quality of evidence: high. Agreement: $100 \%$.

Recommendation $9 b$

We recommend that at least 6 gastric biopsies should be obtained for the diagnosis of $H$ pylori infection during upper endoscopy.
GRADE: Strong recommendation. Quality of evidence: low. Agreement: 93\%.

\section{Practice Points}

1. It is recommended that the initial diagnosis of H pylori infection be performed using invasive gastric biopsy-based methods including the following:

a. Positive bacterial culture $O R$

b. H pylori gastritis on histopathology using the updated Sydney classification with at least 1 other positive test such as RUT, or molecular-based assays where available, including polymerase chain reaction (PCR), or fluorescent in situ hybridization.

2. The initial diagnosis of $H$ pylori infection should not be based on noninvasive tests (ie, ${ }^{13} \mathrm{C}$-UBT, H pylori stool antigen test) or other noninvasive methods. A positive noninvasive test, however, supports the diagnosis in cases in which positive histology is the only invasive test available.

3. At least 6 gastric biopsies should be taken for the initial diagnosis of H pylori infection. Two biopsies should be obtained from the antrum and 2 biopsies from the corpus for the histopathological evaluation applying the updated Sydney classification (48), at least 1 biopsy from the antrum and 1 from the corpus for culture (if available) and at least 1 biopsy for any additional diagnostic tests from the antrum (rapid-urease, or molecular-based assays).

4. In the course of upper gastrointestinal bleeding, false-negative results of gastric biopsy-based methods can occur, that is, histopathology, culture, or RUT. Molecular diagnostic methods may have better test accuracy in this setting $(49,50)$.

5. The transport of biopsies in special transport media rather than normal saline is highly recommended to enhance the success rate for culture.

\section{COMMENT:}

These recommendations remain unchanged from previous guidelines. The current reference "criterion standard" for investigating $H$ pylori-associated disease in children remains upper gastrointestinal endoscopy with biopsies for culture, histology, and RUT (51). None of the available diagnostic tests have $100 \%$ accuracy. Bacterial culture is, however, $100 \%$ specific. The positive predictive value of a diagnostic test rapidly decreases if the prevalence of the infection in the population is low, which is the situation in children in most European and North American countries. Therefore, we recommend that the diagnosis of $H$ pylori infection be based on 2 tests and not only on a positive histology. During the current review period, Seo et al (52) reported the rate of RUT positivity in $255 \mathrm{H}$ pylori-infected children. The authors found that the sensitivity of RUT increased with age (being lowest in the $0-4$-year age group) and with the number of biopsies, whereas the time of an RUT to turn positive took longer in the younger age group. The authors explain their findings by a lower bacterial density in children compared to adolescents and adults. Similar to previous findings, a recent study reported that antral nodularity remains a useful diagnostic predictor of $H$ pylori gastritis in children (53).

\section{Recommendation 10}

We recommend against antibody-based tests (immunoglobulin $\mathrm{G}$ [ $\mathrm{IgG}$ ], IgA) for $H$ pylori in serum, whole blood, urine, and saliva in the clinical setting. 
GRADE: Strong recommendation. Quality of evidence: high. Agreement: $86 \%$.

\section{Practice Points}

1. These antibody-based tests should not be used to detect the presence of active $H$ pylori infection or for use in post-treatment evaluation in children or adolescents.

\section{COMMENT:}

These recommendations remain unchanged from previous guidelines.

\section{Recommendation 11}

We recommend that antimicrobial susceptibility be obtained for the infecting $H$ pylori strain(s) and, the eradication treatment tailored accordingly.

GRADE: Strong recommendation. Quality of evidence: low. Agreement: $86 \%$.

\section{Practice Points}

1. H pylori antimicrobial susceptibility testing should be based on culture biopsy-based methods (E-test or agar dilution) or molecular biopsy-based techniques (real-time PCR, fluorescent in situ hybridization).

2. The transport of biopsies in special transport media will enhance the success rate for culture.

3. Molecular methods, especially commercially available kits based on real-time PCR, are suitable to detect $H$ pylori and clarithromycin resistance in gastric biopsy specimens or gastric juice. At this stage these techniques cannot be recommended for detection of antibiotic resistance in stool.

4. To increase the sensitivity for detecting antimicrobial resistance of $\mathrm{H}$. pylori strains in children colonized with different resistance patterns it is recommended that gastric biopsies for culture-and molecular-based tests be obtained from at least 2 different locations (ie, antrum and body). The biopsies can be sent to the laboratory in the same jar.

\section{COMMENT:}

Antimicrobial resistance patterns vary depending on national/geographic regions and are a major factor in determining the success of eradication therapy (54-61). Treatment failure will result in increased healthcare utilization, and likely puts the child through additional unnecessary procedures and therapies, and the associated risks. Moreover, eradication failure increases the risk of antibiotic resistance of the infecting $H$ pylori strains, thereby reducing the likelihood of successful eradication $(55,56)$. Development of antibiotic resistance may also occur if children are on long-term antibiotics for other comorbidities.

In many countries primary clarithromycin resistance has increased to levels above the recommended threshold $(15 \%)$ for use as a first-line agent for $H$ pylori infection (54). Furthermore, antibiotic resistance rates of $H$ pylori can vary among different regions within the same country (58). Thus, national/regional antibiotic resistance data could be used to guide treatment regimens for $H$ pylori infection (54). In fact, increasing antimicrobial resistance likely explains the reduction in efficacy of clarithromycin containing regimens over time. Resistance to metronidazole has also increased in some populations and regions $(55,60)$. In addition, multiple strains with different antibiotic resistance profiles can exist within the same individual (62). In adults, treatment tailored to antimicrobial susceptibility reduces the risk of treatment failure compared with empirical triple therapy (63). Few comparative data including only a small number of children exist in the pediatric literature.

\section{Recommendation 12}

We recommend that the effectiveness of first-line therapy be evaluated in national/regional centers. GRADE: Strong recommendation. Quality of evidence: low. Agreement: $100 \%$.

\section{Practice Points}

1. As antimicrobial susceptibility testing is not available in all centers, we propose that national/regional effectiveness of $H$ pylori eradication regimens in children and adolescents be evaluated if possible.

\section{COMMENT:}

Treatment failure of $H$ pylori eradication regimens in routine practice is often associated with inappropriate choice of treatment regimen, poor adherence, and antimicrobial resistance. To avoid further investigations, and induction of secondary resistance in the infecting $H$ pylori strains, a primary success rate for eradication should be more than $90 \%$ in per-protocol analysis (54). This goal is not achieved in most current published treatment trials in children (63-68). Therefore, benchmarking is a necessity to assess the local performance of the prescribed regimens and to minimize the risk of treatment failure. This is of particular importance in areas in which antimicrobial susceptibility testing is not available.

\section{Recommendation 13}

We recommend that the physician explain to the family the importance of adherence to the anti$H$ pylori therapy to enhance successful eradication. GRADE: Strong recommendation. Quality of evidence: moderate. Agreement: $86 \%$.

\section{Practice Points}

1. Patient information leaflets with individualized schedules for drug intake may improve adherence.

\section{COMMENT:}

Lack of adherence is a significant risk factor for failure of therapy (6). A recent study published outside of the review period demonstrated that a high success rate for eradication therapy was achieved only in the subset of infected children who took at least $90 \%$ of the prescribed eradication therapy (67). Recommended eradication protocols are composed of at least 3 drugs given for 10 to 14 days. In addition, therapy-related adverse effects, although mostly minor, are quite frequent. Therefore, a proper and detailed explanation of the recommended therapy, potential side effects, and importance of adherence to parents and children is critical to the success of eradication therapy. 


\section{Recommendation 14}

We recommend the following as first-line therapy for $H$ pylori infection as outlined in Table 2.

GRADE: Strong. Quality of evidence: moderate to low for suggested regimens; low for duration; Agreement: $100 \%$.

\section{Practice Points}

1. If the strain is susceptible to clarithromycin (CLA) and to metronidazole (MET), triple therapy (PPI, amoxicillin [AMO], $C L A)$ for 14 days is the preferred choice. In comparison with sequential therapy, this regimen will not induce resistance to metronidazole. In case of treatment failure with PPI, AMO, and CLA a switch to PPI, AMO, and MET can be made without further susceptibility testing.

2. Sequential therapy for 10 days (PPI with AMO for 5 days followed by PPI with CLA and MET for 5 days with doses given in Table 3) is equally effective in patients infected with fully susceptible strains. It, however, has the disadvantage of exposing the child to 3 different antibiotics. Sequential therapy should not be given if the strain is resistant to MET or CLA, or if susceptibility testing is not available. The most recent adult guidelines recommend against the use of sequential therapy as first- or second-line therapy $(69,70)$.

3. Doses of PPI and antibiotics should be calculated based on the bodyweight as shown in Table 3.

4. A higher degree of acid suppression improves the success rate of amoxicillin- and clarithromycin-based therapy. Younger children need a higher PPI dose per $\mathrm{kg}$ bodyweight compared to adolescents and adults to obtain sufficient acid suppression.

5. Esomeprazole and rabeprazole are less susceptible to degradation by rapid metabolizers with CYP2C19 genetic polymorphism, and therefore, may be preferred when available. Rapid metabolizers are more frequent in the Caucasian population $(56 \%-81 \%)$ compared to Asians.

6. The PPI dose given in the table refers to esomeprazole and omeprazole and should be adapted if other PPIs are used. PPIs should be preferentially given at least 15 minutes before meal.

7. For children younger than 8 years, bismuth quadruple therapy refers to bismuth, PPI, AMO, and MET. In children older than 8 years, bismuth quadruple therapy refers to bismuth, PPI, MET, and tetracycline.

8. The routine addition of either single or combination probiotics to eradication therapy to reduce side effects and/or improve eradication rates is not supported by current evidence.

\section{COMMENT:}

Treatment success is negatively affected if the strain is resistant to one of the antibiotics used. Thus recommended therapies should be based on knowledge of antibiotic susceptibility testing (Fig. 1). If primary culture and susceptibility testing is not available, then clarithromycin-based triple therapy should not be used as part of first-line therapy due to high rates of clarithromycin resistance rates worldwide (55-61).

The previous optimism for the use of 10-day sequential therapy (PPI with AMO for 5 days followed by PPI with CLA and MET for 5 days) in treatment naïve patients has now been questioned by additional studies in both children (64-66) and adults (68). A meta-analysis of pediatric studies concluded that a 10-day sequential therapy was superior to 7-day standard triple therapy (relative risk 1.17, 95\% confidence interval: 1.071.28), but

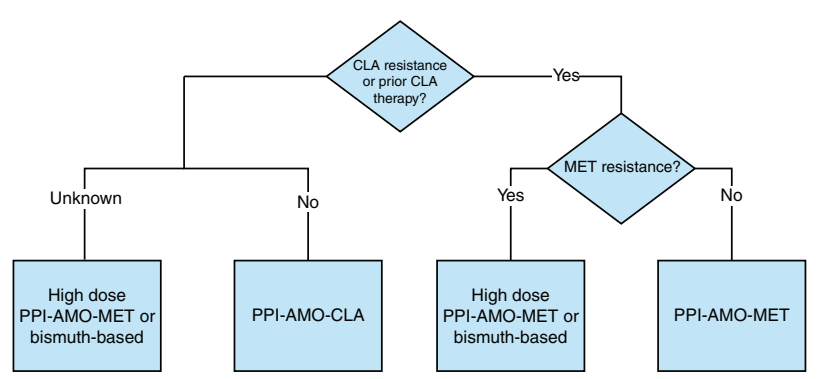

FIGURE 1. Algorithm for selection of eradication therapy for $\mathrm{H}$ pylori infection in children based on knowledge of antibiotic susceptibility. $\mathrm{PPI}=$ proton pump inhibitor.

performed similar to 10- or 14-day triple therapy (65). In an open label, prospective multicenter European study of 165 children, eradication rates with sequential therapy were only $56 \%$ when clarithromycin resistance was present (64). Thus, current evidence indicates that sequential therapy should not be recommended in treatment-naïve children when antibiotic susceptibility of the infecting strain is not known. A recently published European pediatric study (67) that was outside the time frame of our review and not included in our analysis, lends support to the recommendation above. In the present study, primary eradication rates with a high-dose sequential 10-day regimen in children with strains susceptible to both clarithromycin and metronidazole were $85.8 \%$ in the intention to treat analysis. In the presence of metronidazole or clarithromycin resistance these rates, however, dropped to $73.3 \%$ and $71.1 \%$, respectively. In addition to antibiotic resistance, the other major factor affecting eradication in the present study was drug intake of $<90 \%$ of the prescribed medication dose. Although there are no specific pediatric studies addressing this therapy, a 14-day tailored triple therapy is recommended when $H$ pylori stains are resistant to 1 antimicrobial agent (eg, clarithromycin or metronidazole).

There are no new well-designed pediatric studies regarding the use of bismuth in eradication protocols since the last guidelines. One retrospective study in Korean children compared eradication rates with bismuth-based quadruple therapy including omeprazole, amoxicillin, and metronidazole for 7 days with standard triple therapy consisting of omeprazole, amoxicillin, and clarithromycin for 14 days (71). Bismuth-based quadruple therapies had higher eradication rates than standard triple therapy, particularly in the more recent era of 2010 to 2012 . Recent meta-analyses in adult subjects indicate that bismuth-based therapy is effective (68). Thus, based on previous literature and adult studies we recommend bismuth quadruple therapy (where available) in children if $H$ pylori antimicrobial susceptibility is unknown or in the setting of dual resistance to clarithromycin and metronidazole. The other recommended therapy in this setting is high-dose amoxicillin triple therapy with metronidazole (Table 4). The evidence supporting this regimen is, however, limited. In 1 small open multicenter European study of children infected with clarithromycin- and metronidazole-resistant strains, eradication rates using high-dose amoxicillin and PPI with metronidazole was $66 \%$ in intention to treat analysis (72).

In adult patients, a recent meta-analysis showed that concomitant quadruple therapy (PPI and amoxicillin, metronidazole, and clarithromycin given for 10 to 14 days) was one of the most effective treatments, with a high eradication rate and acceptable frequency of adverse events (73). The most recent adult international practice guidelines recommend concomitant therapy as a first-line option $(69,70)$. Currently no studies assessing concomitant 
therapy in the pediatric setting are available for comparison. Future studies should assess this therapy in children and adolescents. In children with primary double resistance to clarithromycin and metronidazole, concomitant therapy may be a better option compared to triple therapy with high-dose amoxicillin.

There are limited well-designed studies in children and adolescents with respect to the optimal duration of anti-H pylori therapy. Regardless of the length of therapy, the choice of antibiotics or the dosing schedule, eradication rates for most anti- $H$ pylori therapies are less than the recommended $90 \%$ per protocol treatment success. Meta-analyses of optimal duration of $H$ pylori eradication therapy in adults have been performed and show that increasing the duration of therapy enhances eradication rates (74). With respect to triple therapy, a recent systematic review and network analysis of studies in adults showed that 14-day duration of treatment improves eradication rates compared to 10-day, and both are superior to 7-day treatment (68). Of note, longer duration therapies were associated with increased rate of adverse events in the present study.

The role of supplemental probiotic therapy to reduce adverse effects, improve adherence, and, thereby, increase efficacy of $H$ pylori eradication regimens remains controversial. Several metaanalyses have attempted to address this (75-77). Although these studies suggest that there may be a benefit for reduction of adverse effects and improved efficacy when added to $H$ pylori eradication therapy, the studies included in these meta-analyses employ different probiotic strains and concentrations making it difficult to draw meaningful conclusions. Therefore, additional sufficiently powered pediatric studies with specific probiotic strains should be performed to develop more reliable conclusions about the benefit of probiotics in $H$ pylori eradication regimens.

There is no evidence to suggest that testing and treating family members for $H$ pylori infection reduces the risk of reinfection. Of note, recent vaccine trials suggest that in areas with high endemic rates of infection, infection rates are low in children older than 6 years $(4)$.

\section{Recommendation $15 a$}

We recommend that the outcome of anti-H pylori therapy be assessed at least 4 weeks after completion of therapy.

GRADE: strong recommendation. Quality of evidence: moderate. Agreement: $100 \%$.

Recommendation $15 \mathrm{~b}$

We recommend that one of the following tests be used to determine whether $H$ pylori treatment was successful:

(1) The ${ }^{13} \mathrm{C}-\mathrm{UBT}$.

(2) A 2-step monoclonal stool $H$ pylori antigen test. GRADE: Strong recommendation. Quality of evidence: high. Agreement: $93 \%$.

\section{Practice Points}

1. The relief of symptoms is not an indicator for successful treatment. Therefore, all children treated for $\mathrm{H}$ pylori should be assessed for treatment success with a reliable test.

2. Endoscopy and biopsy-based tests to confirm eradication are rarely needed in pediatric patients with uncomplicated PUD. Gastric and duodenal peptic ulcers have a low risk for relapse with clearance of the infection.
3. The ${ }^{13} \mathrm{C}$-UBT may give false-positive results in children younger than 6 years of age because of the lower distribution volume and different $\mathrm{CO}_{2}$ production rate (78-80). Falsepositive results may also be due to technical difficulties in performing ${ }^{13} \mathrm{C}$-UBT in young children because they are often unwilling to swallow the substrate and oral urease-producing organisms can then split the substrate. This has little relevance in clinical practice in which eradication therapy is, however, rarely indicated in children younger than 6 years.

4. The use of ${ }^{14} \mathrm{C}-U B T$ is not recommended in children.

5. The accuracy of the 2-step monoclonal stool antigen test is comparable to the ${ }^{13} \mathrm{C}$-UBT and performance is unrelated to age. This test is not available (licensed) in North America.

6. The polyclonal stool antigen test has a lower accuracy compared to the ${ }^{13} \mathrm{C}$-UBT and the monoclonal stood test, but may be an option in situations when neither of the other 2 tests are available $(81,82)$.

7. Rapid office-based stool tests have a lower performance compared to laboratory-based tests.

\section{COMMENT:}

When considering a test for cure, it is important to employ the most accurate test for active infection (78). In most clinical settings, noninvasive testing, either ${ }^{13} \mathrm{C}-\mathrm{UBT}$ or fecal antigen testing should be employed to ensure cure of infection has been achieved (78). The ${ }^{13} \mathrm{C}$-UBT is well validated against invasive tests and performs well even with a variety of test protocols $(79,80)$. In contrast, fecal tests depend on the antigen used and may differ in their performance $(81-86)$. For both ${ }^{13} \mathrm{C}-\mathrm{UBT}$ and fecal antigen testing, false-negative results can occur when medications are taken that decrease the bacterial load or suppress gastric acid (78). Therefore testing for cure should be performed at least 4 weeks after completion of eradication therapy to avoid falsenegative results.

\section{Recommendation 16}

We recommend that when $H$ pylori treatment fails, rescue therapy should be individualized considering antibiotic susceptibility, the age of the child, and available antimicrobial options.

GRADE: Strong recommendation. Quality of evidence: low. Agreement: 93\%.

\section{Practice Points}

1. See Table 5 for specific recommendations for rescue therapy.

\section{COMMENT:}

In comparison with adults, reduced options for rescue therapy exist in children, further emphasizing the need for the selection of initial regimens with the best chance of success. In patients who have previously failed $H$ pylori eradication therapy, the choice of rescue therapy should consider initial antibiotic susceptibility status (if known) and the first-line regimens employed (Table 5). Where possible, rescue therapy should be based on antibiotic resistance profiles. Antibiotics with a high likelihood of the infecting strain to develop secondary resistance (eg, clarithromycin, metronidazole) should be avoided if they were employed in the initial regimen. Studies in adults suggest that increasing acid suppression and metronidazole dose may improve 
efficacy of eradication therapy (87). Although there are no data addressing this issue for rescue therapy in children, employing higher dosages of PPI and metronidazole may be considered. As described in recommendation 14, 1 study showed that in children infected with clarithromycin- and metronidazole-resistant strains, the eradication rate using high dose amoxicillin and PPI with metronidazole was $66 \%$ in intention to treat analysis (72). In a recent multicenter, open-label, single-arm, clinical trial (82) of $H$ pylori-positive adults who had failed more than one previous course of omeprazole-amoxicillin-clarithromycin therapy, a quadruple regimen of bismuth, metronidazole, and tetracycline plus omeprazole produced a high eradication rate (up to $95.8 \%$ ). As expected, these authors concluded that bismuth-based regimen offers an effective option as rescue therapy (88).

\section{SUMMARY}

Because of the unacceptably low $H$ pylori eradication rates in children reported in the literature from different countries, and the increasing rates of antibiotic resistance, these guidelines now focus on prescribing treatment for $H$ pylori in children only when it is clinically indicated, or when following discussion with parents it is deemed appropriate to treat an incidental finding of $H$ pylori in children (Table 6). Previous guideline recommendations of triple or sequential therapy for treatment-naïve $H$ pylori-infected children and adolescents can no longer be supported. Instead, to achieve an initial eradication success rate of $90 \%$ and higher, therapy should be based on knowledge of antibiotic resistance profiles and therapy tailored accordingly using sufficiently high doses and treatment durations of 10 to 14 days. The importance of adherence for optimal successful eradication should be emphasized to the parents and child. Clarithromycin-containing regimens should be restricted to those infected with clarithromycin-susceptible strains. When antibiotic susceptibility profiles are not known, high-dose triple therapy with PPI, amoxicillin, and metronidazole for 14 days is recommended as first-line therapy. Bismuth-based quadruple therapy can also be considered for first-line therapy in countries where it is licensed for use in children. As with the previous guidelines, testing for cure should be performed with either the $C^{13}$-UBT or a fecal antigen test at least 4 weeks after therapy to avoid false-negative results. Second-line therapies present a real challenge in children due to the limited number of antibiotics that are appropriate to use in children. Second-line therapies should take into consideration the antibiotics employed for the initial therapy, duration of treatment, and, where possible, should be based on antibiotic resistance profiles.

\section{Future Directions for the Management of $H$ pylori-infected Children}

Future studies should focus on obtaining antibiotic resistance rates across regions to help direct optimal therapy. In addition, highquality studies evaluating novel approaches and therapies in children including quadruple concomitant therapy (PPI and amoxicillin, metronidazole, and clarithromycin given for 10-14 days), bismuth-based regimens, and the role of supplemental probiotics are needed. Importantly, studies of prevention of infection with optimization of vaccine strategies should be performed.

TABLE 6. Highlights of changes in new guidelines in comparison to the previous guidelines

New recommendations

Previous recommendations

1. Who to test for $H$ pylori infection

Testing for $H$ pylori during endoscopy should only be performed if intending to treat

Testing for $H$ pylori is not recommended in the initial investigation of iron deficiency anemia

Weak recommendation to consider noninvasive testing for $H$ pylori infection in the setting of chronic ITP

Recommend against testing in the setting of short stature

Removal of recommendation for testing in the setting of family history of gastric cancer or MALT, as rarely encountered

2. How to test for $H$ pylori

Six gastric biopsies for either (a) histopathology (H pylori-positive gastritis) plus at least 1 other positive biopsy-based test or (b) positive culture.

3. Who to treat with $H$ pylori infection

If $H$ pylori is an incidental finding at endoscopy treatment may be considered following careful discussion of the risks and benefits of $H$ pylori treatment with the patient/parents

Weak recommendation for patient with chronic ITP

4. How to treat infected patients

Evaluation of effectiveness of first-line therapy in national/regional centers

Differences in first-line recommended therapies and dosing (see Table 2 and algorithm)

Tailored treatment based on antibiotic sensitivity where available.

Duration of therapy 14 days

Higher dose of PPI, particularly in younger children, with doses from 1.5 to $2.5 \mathrm{mg} /$ $\mathrm{kg}$ bodyweight and day

Recommendation that physician explain to the patient/ family the importance of adherence

\author{
Not addressed \\ Not addressed \\ Insufficient evidence that infection is causally related to ITP \\ Insufficient evidence that infection is causally related to short \\ stature \\ Consider testing in the setting of family history of gastric \\ cancer or MALT
}

Biopsies from corpus and antrum for histopathology plus rapid urease test or culture

When H pylori is detected by biopsy-based methods in absence of PUD, treatment may be considered

Surveillance of antibiotic resistance rates in different countries First-line therapy options: standard triple,

bismuth + amoxicillin + imidazole or sequential

In areas with high clarithromycin resistance, perform susceptibility testing for clarithromycin to tailor its use

Duration of therapy 7-14 days

PPI dose ranging from 1 to $2 \mathrm{mg} / \mathrm{kg}$ bodyweight and day

Not a specific recommendation, addressed in discussion

ITP $=$ immune thrombocytopenic purpura; MALT $=$ mucosa-associated lymphoid tissue; PPI $=$ proton pump inhibitor; PUD $=$ peptic ulcer disease. 
ESPGHAN and NASPGHAN are not responsible for the practices of health care providers and provide guidelines and position papers as indicators of best practice only. Diagnosis and treatment are at the discretion of physicians.

\section{REFERENCES}

1. Amieva M, Peek RM Jr. Pathobiology of Helicobacter pyloriassociated gastric cancer. Gastroenterology 2016;150:64-78.

2. Sierra MS, Hastings EV, Goodman KJ. What do we know about benefits of $H$. pylori treatment in childhood? Gut Microbes 2013;4: 549-67.

3. Spee LAA. Association between Helicobacter pylori and gastrointestinal symptoms in children. Pediatrics 2010;125:e651-69.

4. Zeng M, Mao XH, Li JX, et al. Efficacy, safety, and immunogenicity of an oral recombinant Helicobacter pylori vaccine in children in China: a randomised, double-blind, placebo-controlled, phase 3 trial. Lancet 2015;386:1457-64.

5. Graham DY, Fischbach L. Helicobacter pylori treatment in the era of increasing antibiotic resistance. Gut 2010;59:1143-53.

6. Koletzko S, Jones NL, Goodman KJ, et al. Evidence-based guidelines from ESPGHAN and NASPGHAN for Helicobacter pylori infection in children. J Pediatr Gastroenterol Nutr 2011;53:230-43.

7. Guyatt GH, Oxman AD, Vist GE, et al. GRADE: an emerging consensus on rating quality of evidence and strength of recommendations. $B M J$ 2008;336:924-6.

8. Harris PR, Wright SW, Serrano C, et al. Helicobacter pylori gastritis in children is associated with a regulatory T-cell response. Gastroenterology 2008;134:491-9.

9. Melo FF, Rocha AM, Rocha GA, et al. A regulatory instead of an IL-17 T response predominates in Helicobacter pylori-associated gastritis in children. Microb Infect 2012;14:341-7.

10. Sivapalasingam S, Rajasingham A, Macy JT, et al. Recurrence of Helicobacter pylori infection in Bolivian children and adults after a population-based "screen and treat" strategy. Helicobacter 2014;19: 343-8.

11. Morgan DR, Torres J, Sexton R, et al. Risk of recurrent Helicobacter pylori infection 1 year after initial eradication therapy in 7 Latin American communities. JAMA 2013;309:578-86.

12. Feydt-Schmidt A, Kindermann A, Konstantopoulos N, et al. Reinfection rate in children after successful Helicobacter pylori eradication. Eur J Gastroenterol Hepatol 2002;14:1119-23.

13. Leontiiadis G, Ford AC. Helicobacter pylori eradication: gastric cancer prevention. Systematic review 406. BMJ Clin Evid 2015;12:406http:// clinicalevidence.bmj.com.myaccess.library.utoronto.ca/x/systematicreview/0406/overview.html. 2015. Accessed January 8, 2016.

14. Blaser MJ, Chen Y, Reibman J. Does Helicobacter pylori infection protect against asthma and allergy? Gut 2008;57:561-7.

15. Serrano CA, Talesnik E, Pena A, et al. Inverse correlation between allergy markers and Helicobacter pylori infection in children is associated with elevated levels of TGF-beta. Eur J Gastroenterol Hepatol 2011;23:656-63.

16. Zevit N, Balicer RD, Cohen HA, et al. Inverse association between Helicobacter pylori and pediatric asthma in a high-prevalence population. Helicobacter 2011;17:30-5.

17. Amberbir A, Medhin G, Erku W, et al. Effect of helicobacter, geohelminth infection and selected commensal bacteria on the risk of allergic disease and sensitization in 3-year-old Ethiopian children. Clin Exp Allergy 2011;41:1422-30.

18. Arnold IC, Hitzler I, Müller A. The immunomodulatory properties of Helicobacter pylori confer protection against allergic and chronic inflammatory disorders. Front Cell Infect Microbiol 2012;2:1-11.

19. Bontems P, Kalach N, Vanderpas J, et al. Helicobacter pylori Infection in European children with gastro-duodenal ulcers and erosions. Pediatr Infect Dis $J$ 2013;32:1324-9.

20. Hyams JS, Di Lorenzo C, Saps M, et al. Functional disorders: children and adolescents. Gastroenterology 2016 [Epub ahead of print].

21. Emiroglu HH. Is there a relationship between Helicobacter pylori infection and erosive reflux disease in children? Acta Padiatrica 2010;99:121-5.
22. Llanes R, Millan LM, Escobar MP, et al. Low prevalence of Helicobacter pylori among symptomatic children from a hospital in Havana, Cuba. J Trop Pediatr 2012;58:231-4.

23. Mansour MM, Al Hadidi KhM, Omar MA. Helicobacter pylori and recurrent abdominal pain in children: is there any relation? Trop Gastroenterol 2012;33:55-61.

24. Ganesh M, Nurko S. Functional dyspepsia in children. Pediatr Ann 2014:43:e101-5.

25. Thakkar K, Chen L, Tessier ME, et al. Outcomes of children after esophagogastroduodenoscopy for chronic abdominal pain. Clin Gastroenterol Hepatol 2014;12:963-9.

26. Dhroove G, Chogle A, Saps M. A million-dollar work-up for abdominal pain: is it worth it? J Pediatr Gastroenterol Nutr 2010;51:579-83.

27. Thompson J, Biggs BA, Pasricha SR. Effects of daily iron supplementation in 2- to 5-year-old children: systematic review and meta-analysis. Pediatrics 2013;131:739-53.

28. WHO. Guideline: Intermittent Iron Supplementation in Preschool and School-age Children Geneva: World Health Organization; 2011.

29. Muhsen K, Nir A, Spungin-Bialik A, et al. Interaction among ethnicity, socioeconomic status, and Helicobacter pylori seroprevalence in Israeli children and adolescents. J Pediatr Gastroenterol Nutr 2011;53:524-7.

30. Bauer S, Krumbiegel P, Richter M, et al. Influence of sociodemographic factors on Helicobacter pylori prevalence variability among schoolchildren in Leipzig, Germany. A long-term follow-up study. Cent Eur J Public Health 2011;19:42-5.

31. Janjetic MA, Goldman CG, Balcarce NE, et al. Iron, zinc, and copper nutritional status in children infected with Helicobacter pylori. J Pediatr Gastroenterol Nutr 2010;51:85-9.

32. Mendoza E, Camorlinga-Ponce M, Perez-Perez G, et al. Present and past Helicobacter pylori infection in Mexican school children. Helicobacter 2014;19:55-64.

33. Pacey A, Weiler H, Egeland GM. Low prevalence of iron-deficiency anaemia among Inuit preschool children: Nunavut Inuit Child Health Survey, 2007-2008. Public Health Nutr 2011;14:1415-23.

34. Thankachan P, Muthayya S, Sierksma A, et al. Helicobacter pylori infection does not influence the efficacy of iron and vitamin $\mathrm{B}(12)$ fortification in marginally nourished Indian children. Eur J Clin Nutr 2010;64:1101-7.

35. Gonzalez- Lopez MA, Velazquez-Guardarrama N, Romero-Espejel $\mathrm{ME}$, et al. Heliocbacter pylori secretes the chaperonin GroEL (HSP60) which binds iron. FEBS Lett 2013;587:1823-8.

36. Xia W, Zhang X, Wang J, et al. Survey of anaemia and Helicobacter pylori infection in adolescent girls in Suihua, China and enhancement of iron intervention effects by H. pylori eradication. Br J Nutr 2012;108: 357-62.

37. Ferrara M, Capozzi L, Russo R. Effect of Helicobacter pylori eradication on platelet count in children with chronic idiopathic thrombocytopenic purpura. Hematology 2009;14:282-5.

38. Russo G, Miraglia V, Branciforte F, et al. Effect of eradication of Helicobacter pylori in children with chronic immune thrombocytopenia: a prospective, controlled, multicenter study. Pediatr Blood Cancer 2011;56:273-8.

39. Brito HS, Braga JA, Loggetto SR, et al. Helicobacter pylori infection and immune thrombocytopenic purpura in children and adolescents: a randomized controlled trial. Platelets 2015;26:336-41.

40. Vilchis J, Duque X, Mera R, et al. Association of Helicobacter pylori infection and height of Mexican children of low socioeconomic level attending boarding schools. Am J Trop Med Hyg 2009;81:1091-6.

41. Gulcan M, Ozen A, Karalepe HO, et al. Impact of H. pylori on growth: is the infection or mucosal disease related to growth impairment? Dig Dis Sci 2010;55:2878-86.

42. Ozen A, Furman A, Berber M, et al. The effect of Helicobacter pylori and economic status on growth parameters and leptin, ghrelin, and insulin-like growth factor (IGF)-I concentrations in children. Helicobacter 2011;16:55-65.

43. Kwiecien J, Ziora K, Oswiecimska J, et al. Prevalence of Helicobacter pylori in children with growth deficiency. Pediatria Wspolczesna 2010;12:2010.

44. Yang YJ, Sheu BS, Yang HB, et al. Eradication of Helicobacter pylori increases childhood growth and serum acylated ghrelin levels. World $J$ Gastroenterol 2012;18:2674-81. 
45. Goodman KJ, Correa P, Mera R, et al. Effect of Helicobacter pylori infection on growth velocity of school-age Andean children. Epidemiology 2011;22:118-26.

46. Mera RM, Bravo LE, Goodman KJ, et al. Long-term effects of clearing Helicobacter pylori on growth in school-age children. Pediatr Infect Dis J 2012;31:263-6.

47. Calvet X. Diagnosis of Helicobacter pylori infection in the proton pump inhibitor era. Gastroenterol Clin North Am 2015;44:507-18.

48. Dixon MF, Genta RM, Yardley JH, et al. Classification and grading of gastritis. The updated Sydney System. International Workshop on the Histopathology of Gastritis, Houston 1994. Am J Surg Pathol 1996;20:1161-81.

49. Rimbara E, Sasatsu M, Graham DY. PCR detection of Helicobacter pylori in clinical samples. Methods Mol Biol 2013;943:279-87.

50. Saez J, Belda S, Santibanez M, et al. Real-time PCR for diagnosing Helicobacter pylori infection in patients with upper gastrointestinal bleeding: comparison with other classical diagnostic methods. J Clin Microbiol 2012;50:3233-7.

51. Crowley E, Bourke B, Hussey S. How to use Helicobacter pylori testing in paediatric practice. Arch Dis Child Educ Pract Ed 2013;98:18-25.

52. Seo JH, Park JS, Yeom JS, et al. Correlation between positive rate and number of biopsy samples on urease test in childhood Helicobacter pylori infection. J Korean Med Sci 2014;29:106-9.

53. Hidaka N, Nakayama Y, Horiuchi A, et al. Endoscopic identification of Helicobacter pylori gastritis in children. Dig Endosc 2010;22:90-4.

54. Graham DY, Lee Y-C, Wu M-S. Rational Helicobacter pylori therapy: evidence-based medicine rather than medicine-based evidence. Clin Gastroenterol Hepatol 2014;12:177-86.

55. Raymond J, Lamarque D, Kalach N, et al. High level of antimicrobial resistance in French Helicobacter pylori isolates. Helicobacter 2010;15:21-7.

56. Megraud F, Coenen S, Versporten A, et al. Helicobacter pylori resistance to antibiotics in Europe and its relationship to antibiotic consumption. Gut 2013;62:34-42.

57. Miendje Deyi VY, Bontems P, Vanderpas J, et al. Multicenter survey of routine determinations of resistance of Helicobacter pylori to antimicrobials over the last 20 years (1990 to 2009) in Belgium. J Clin Microbiol 2011;49:2200-9.

58. Seo JH, Jun JS, Yeom JS, et al. Changing pattern of antibiotic resistance of Helicobacter pylori in children during 20 years in Jinju, South Korea. Pediatr Int 2013;55:332-6.

59. Nguyen TV, Bengtsson C, Yin L, et al. Eradication of Helicobacter pylori in children in Vietnam in relation to antibiotic resistance. Helicobacter 2012;17:319-25.

60. Zevit N, Levy I, Shmuely H, et al. Antibiotic resistance of Helicobacter pylori in Israeli children. Scand J Gastroenterol 2010;45:550-5.

61. Oleastro M, Cabral J, Ramalho PM, et al. Primary antibiotic resistance of Helicobacter pylori strains isolated from Portuguese children: a prospective multicentre study over a 10 year period. J Antimicrob Chemother 2011;66:2308-11.

62. Selgrad M, Tammer I, Langner C, et al. Different antibiotic susceptibility between antrum and corpus of the stomach, a possible reason for treatment failure of Helicobacter pylori infection. World J Gastroenterol 2014;20:16245-51.

63. Wenzhen Y, Yumin L, Quanlin G, et al. Is antimicrobial susceptibility testing necessary before first-line treatment for Helicobacter pylori infection? Meta-analysis of randomized controlled trials. Intern Med 2010;49:1103-9.

64. Bontems P, Kalach N, Oderda G, et al. Sequential therapy versus tailored triple therapies for Helicobacter pylori infection in children. J Pediatr Gastroenterol Nutr 2011;53:646-50.

65. Horvath A, Dziechciarz P, Szajewska H. Meta-analysis: sequential therapy for Helicobacter pylori eradication in children. Aliment Pharmacol Ther 2012;36:534-41.

66. Horvath A, Dziechciarz P, Szajewska H. Letter: sequential therapy for Helicobacter pylori eradication in children updated meta-analysis of randomized controlled trials. Aliment Pharmacol Ther 2013;37:835-6.

67. Schwarzer A, Bontems P, Urruzuno P, et al. Sequential Therapy for Helicobacter pylori Infection in Treatment-naive Children. Helicobacter 2016;21:106-13.
68. Li BZ, Threapleton DE, Wang JY, et al. Comparative effectiveness and tolerance of treatments for Helicobacter pylori: systematic review and network meta-analysis. BMJ 2015;351:h4052.

69. Fallone CA, Chiba N, Veldhuyzen van Zanten S, et al. Clinical practise guidelines for the treatment of Helicobacter pylori infection: the Toronto Consensus. Gastroenterology 2016 [Epub ahead of print].

70. Malfertheiner P, Megraud F, O'Morain CA, et al. Management of Helicobacter pylori infection-the Maastricht V/Florence Consensus Report. Gut 2017;66:6-30.

71. Hong J, Yang HR. Efficacy of proton pump inhibitor-based triple therapy and bismuth-based quadruple therapy for Helicobacter pylori eradication in Korean children. Pediatr Gastroenterol Hepatol Nutr 2012; 15:237-42.

72. Schwarzer A, Bontems P, Urruzuno P, et al. New effective treatment regimen for children infected with a double-resistant Helicobacter pylori strain. J Pediatr Gastroenterol Nutr 2011;52:424-8.

73. Gisbert JP, McNicholl AG. Eradication of Helicobacter pylori infection with non-bismuth quadruple concomitant therapy. In: Talebi Bezmin Abadi, A. ed. Helicobacter pylori: to be or not to be! Sharjah, UAE: Bentham; 2016.

74. Yuan Y, Ford AC, Khan KJ, et al. Optimum durations of regimens for Helicobacter pylori eradication. Cochrane Database Syst Rev 2013:CD008337.

75. Li S, Huang XL, Sui JZ, et al. Meta-analysis of randomized controlled trials on the efficacy of probiotics in Helicobacter pylori eradication therapy in children. Eur J Pediatr 2014;173:153-61.

76. Zheng X, Lyu L, Mei Z. Lactobacillus-containing probiotic supplementation increases Helicobacter pylori eradication rate: evidence from a meta-analysis. Rev Esp Enferm Dig 2013;105:445-53.

77. Szajewska H, Horvath A, Piwowarczyk A. Meta-analysis: the effects of Saccharomyces boulardii supplementation on Helicobacter pylori eradication rates and side effects during treatment. Aliment Pharmacol Ther 2010;32:1069-79.

78. Guarner J, Kalach N, Elitsur Y, et al. Helicobacter pylori diagnostic tests in children: review of the literature from 1999 to 2009. Eur J Pediatr 2010;169:15-25.

79. Leal YA, Flores LL, Fuentes-Panana EM, et al. 13C-urea breath test for the diagnosis of Helicobacter pylori infection in children: a systematic review and meta-analysis. Helicobacter 2011;16:327-37.

80. Pacheco SL, Ogata SK, Machado RS, et al. Diagnosis of Helicobacter pylori infection by means of reduced-dose (1)(3)C-urea breath test and early sampling of exhaled breath. J Pediatr Gastroenterol Nutr 2013;57:607-11.

81. Leal YA, Cedillo-Rivera R, Simon JA, et al. Utility of stool samplebased tests for the diagnosis of Helicobacter pylori infection in children. J Pediatr Gastroenterol Nutr 2011;52:718-28.

82. Zhou X, Su J, Xu G, et al. Accuracy of stool antigen test for the diagnosis of Helicobacter pylori infection in children: a meta-analysis. Clin Res Hepatol Gastroenterol 2014;38:629-38.

83. Raguza D, Machado RS, Ogata SK, et al. Validation of a monoclonal stool antigen test for diagnosing Helicobacter pylori infection in young children. J Pediatr Gastroenterol Nutr 2010;50:400-3.

84. Okuda M, Osaki T, Kikuchi S, et al. Evaluation of a stool antigen test using a $\mathrm{mAb}$ for native catalase for diagnosis of Helicobacter pylori infection in children and adults. J Med Microbiol 2014;63:1621-5.

85. Queiroz DM, Saito M, Rocha GA, et al. Helicobacter pylori infection in infants and toddlers in South America: concordance between [13C] urea breath test and monoclonal H. pylori stool antigen test. J Clin Microbiol 2013;51:3735-40.

86. Tiryaki Z, Yilmaz-Ciftdogan D, Kasirga E. Diagnostic value of stool antigen and antibody tests for Helicobacter pylori infection in Turkish children with upper gastrointestinal complaints before and after eradication. Turk J Pediatr 2010;52:505-11.

87. Molina-Infante J, Lucendo AJ, Angueira T, et al. Optimised empiric triple and concomitant therapy for Helicobacter pylori eradication in clinical practice: the OPTRICON study. Aliment Pharmacol Ther 2015;41:581-9.

88. Delchier JC, Malfertheiner P, Thieroff-Ekerdt R. Use of a combination formulation of bismuth, metronidazole and tetracycline with omeprazole as a rescue therapy for eradication of Helicobacter pylori. Aliment Pharmacol Ther 2014;40:171-7. 